\title{
Artifactual pyrosequencing reads in multiple-displacement-amplified sediment metagenomes from the Red Sea
}

The Multiple Displacement Amplification (MDA) protocol is reported to introduce different artifacts into DNA samples with impurities. In this study, we report an artifactual effect of MDA with sediment DNA samples from a deep-sea brine basin in the Red Sea. In the metagenomes, we showed the presence of abundant artifactual 454 pyrosequencing reads over sizes of 50 to $220 \mathrm{bp}$. Gene fragments translocated from neighboring gene regions were identified in these reads . Occasionally, the translocation occurred between the gene fragments from different species. Reads containing these gene fragments could form a strong stem-loop structure. More than $60 \%$ of the artifactual reads could fit the structural models. MDA amplification is probably responsible for the massive generation of the artifactual reads with the secondary structure in the metagenomes. Possible sources of the translocations and structures are discussed. 
7

*Corresponding author: boqianpy@ust.hk, Tel: 852-2358-7331

Keywords: gene fragments; MDA; metagenome; artifactual 454 reads

Running title: Artifactual reads in sediment metagenomes

1. Division of Life Science, Hong Kong University of Science and Technology, Clear Water Bay, Hong Kong SAR, China

2. Institute of Oceanography, Chinese Academy of Science, 7 Nanhai Road, Qingdao, China multiple-displacement-amplified sediment metagenomes from the Red Sea

\author{
Yong Wang ${ }^{1}$, On On Lee ${ }^{1}$, Jiang Ke Yang ${ }^{1}$, Tiegang $\mathrm{Li}^{2}$, Pei Yuan Qian ${ }^{1, *}$
}




\section{Abstract}

35 The Multiple Displacement Amplification (MDA) protocol is reported to introduce different 36 artifacts into DNA samples with impurities. In this study, we report an artifactual effect of MDA 37 with sediment DNA samples from a deep-sea brine basin in the Red Sea. In the metagenomes, we showed the presence of abundant artifactual 454 pyrosequencing reads over sizes of 50 to 220 bp. Gene fragments translocated from neighboring gene regions were identified in these reads . Occasionally, the translocation occurred between the gene fragments from different species. Reads containing these gene fragments could form a strong stem-loop structure. More than $60 \%$ of the artifactual reads could fit the structural models. MDA amplification is probably responsible for the massive generation of the artifactual reads with the secondary structure in the metagenomes. Possible sources of the translocations and structures are discussed. 


\section{Introduction}

The development of pyrosequencing techniques has brought unprecedented opportunities to environmental microbiological studies (Logares et al., 2012). Microbial metagenomes from a variety of ecological settings have been obtained and microbial communities in unique habitats are increasingly uncovered by bar-coded pyrosequencing of $16 \mathrm{~S}$ ribosomal RNA amplicons (Biddle et al., 2008; Ferrer et al., 2012; Huse et al., 2008; Smedile et al., 2012). We are able to determine the composition of microbial communities and their roles in elemental cycles by the analyses of pyrosequencing data. Novel genes and pathways involved in new metabolisms and adaptation mechanisms can be predicted and validated in subsequent experiments (Singh et al., 2009). As a result, microbial ecology has rapidly developed in recent years. However, the quality of pyrosequencing on different platforms is still a major concern (Quail et al., 2012). For example, the ROCHE 454 platform shows weakness in deciphering homopolymers, which account for about $40 \%$ of its sequencing errors (Huse et al., 2007). Artifactual duplications represent $11-35 \%$ of the raw reads generated by the 454 platform (Gomez-Alvarez et al., 2009). Moreover, some DNA samples from extreme environments need to be amplified by Whole Genome Amplification (WGA) to meet the minimum requirement for the pyrosequencing (Dean et al., 2002). Enough DNA sample from the bacterial strain of interest in an environmental sample can then be subjected to pyrosequencing, which enables direct assessment of genomes of individual bacteria, and bypasses the isolation and cultivation procedure in the laboratory.

Despite technical improvements, WGA still has many problems in the amplification of small amounts of DNA. As one of the widely used WGA protocols, MDA uses the phi29 DNA polymerase and random primers to amplify DNA templates (Dean et al., 2002; Dean et al., 
2001). The typical process of MDA amplification is illustrated (Fig.1A). It has been successfully used to amplify DNA samples from different small biological specimens (Lasken and Stockwell, 2007; Raghunathan et al., 2005; Zhang et al., 2006). But MDA also introduces problems into the amplified DNA sample. Firstly, amplification bias and errors cannot be avoided. Secondly, undesired background amplification may occur and occasionally occupy about $70 \%$ of the final MDA product (Raghunathan et al., 2005). Therefore, small exogenous DNA contamination and plasmids (amplified as shown Fig.1B) as the major sources of error should be removed from the DNA template (Zhang et al., 2006). Another source of background amplification is template-independent, primer-primer amplification, accounting for up to $75 \%$ of the total yield (Spits et al., 2006). It is intensified by a low concentration of DNA template and exogenous DNA contamination (Pan et al., 2008). This problem has however been recently resolved by using constrained-randomized primers that cannot hybridize with each other(Zhang et al., 2006). Thirdly, chimeras and translocations were frequently identified in MDA amplified samples (Lasken and Stockwell, 2007; Zhang et al., 2006). A report showed that hundreds of chimeras with DNA rearrangements were identified in 454 reads for an Escherichia coli genome from a single cell after MDA amplification (Lasken and Stockwell, 2007). Most of them have a sequence inversion that allows the formation of inverted repeats. The occurrence of chimeric sequences was regarded as a result of the incorrect interaction between nearby concurrently synthesized sequences (Lasken and Stockwell, 2007). Although these chimeras can be identified and filtered later, this finding is a reminder of other unknown problems during the MDA process. Before we can resolve the technical issues completely, conclusions based on the metagenomic analysis must be treated cautiously. Therefore, there is an urgent need to learn about all the weaknesses in sample treatment protocols and pyrosequencing platforms. 
111 Generally, coastal sediments are rich in microbes and therefore a DNA sample extracted with 112 traditional methods is sufficient for pyrosequencing. However, in deep-sea sediments, the 113 bacterial biomass is low due to harsh environments, which necessitates the use of MDA for 114 metagenomic studies in these extreme biospheres. Raw DNA samples extracted from sediments 115 often contain extracellular DNA and plasmids (Pietramellara et al., 2009). The former arises 116 from the lysis of dead cells (Levy-Booth et al., 2007). The presence of the non-genomic DNA 117 will raise the background amplification during MDA amplification. In this study, microbes from 118 a deep-sea saline basin in the Red Sea were studied. Although DNA had been extracted, the amount was not large enough for 454 pyrosequencing. MDA amplification had to be used to 120 amplify the DNA samples. However, in this sediment, extracellular DNA was probably abundant 121 because it can be preserved in the saline anaerobic environment (Borin et al., 2008). On the other 122 hand, the extracellular DNA samples may have stable secondary structures (Fig. 1C-D) to resist 123 degradation naturally (Steinberger and Holden, 2005). The presence of the contaminant in our 124 samples can be used to examine the biasing effects of MDA amplification. We pyrosequenced 125 MDA-amplified DNA samples from five subsuperficial layers in a sediment core. The 126 assessment of the biasing effects can be determined by examining over-abundant genes in pyrosequenced metagenomes. Several genes with abundant short reads in the metagenomes from the deep layers were studied. These reads generally contained two gene regions (gene fragments). Translocations of the gene fragments were identified in the reads and stem-loop structures could be constructed by the translocated subsections, indicating that multiplication of the fragments was probably triggered by the secondary structure. Hence we conclude that the observed abundant short reads are artifacts of the MDA treatment. 


\section{Materials and Methods}

A $2.25-m$ gravity sediment core was obtained from the Atlantis II Deep $\left(21^{\circ} 20.76^{\prime} \mathrm{N}, 38^{\circ} 04.68^{\prime}\right.$ E) in the Red Sea in 2008 (Bower, 2009). Sediment slices of 12-15 cm (Sed12), 63-66 cm (Sed63), 105-108 cm (Sed105), 183-186 cm (Sed183), and 222-225 cm (Sed222) were used for DNA extraction. Ten grams of sediment from the five layers were used for DNA extraction. The crude DNA was purified with an MO BIO Power Max soil DNA isolation kit (Solana Beach, CA, USA). A REPLI-g MDA kit (Qiagen, Hilden, Germany) was applied to amplify the microbial genomic DNA from the sediment layers, followed by pyrosequencing on a ROCHE 454 FLX Titanium platform.

A flowchart of data analysis is illustrated in Figure 1. A protein database was downloaded from the Kyoto Encyclopedia of Genes and Genomes (KEGG, http:/www.genome.jp/kegg, v51). Pyrosequencing reads were used to BLASTX (BLAST2.2.20) against the protein database, with parameters of “-p blastx -e 0.0001-m 8 -Q 11”. Reads for the same KEGG genes were pooled and then sorted into different length ranges in a size increment of $10 \mathrm{bp}$. The percentage of reads in each of the ranges was calculated. The position of the reads aligned on the full length proteins was determined by the above BLASTX results. If the proteins belonged to the same genus, the protein sizes were generally the same. In each section (10 aa) of the protein, the number of the aligned reads was recorded and the percentage of the reads in all those for the gene was calculated. If the reads were derived from more than one genus according to the result of the best BLAST hits, the proteins in the best hits were first subjected to multiple alignment by ClustalW (www.clustal.org) and then the unaligned parts from both ends were trimmed away. The 
155

156

157

158

159

160

161

162

matching positions of the reads on the proteins were then adjusted to those of the trimmed proteins.

After the reads were sorted into different KEGG genes, short $(<220$ bp; see the distribution shown in Fig. 2) and long reads (> $220 \mathrm{bp)}$ were separated and counted as $\mathrm{N}_{<220}$ and $\mathrm{N}_{>220}$. The percentage of the short reads $\left(\mathrm{P}_{<220}\right)$ in a gene was then calculated. An interesting observation was that a majority of these short reads did not have a full-length alignment with a reference protein by the BLAST search. This means that a small part of them could not be matched to known genes under the current searching criteria. Start and stop points in the alignments were then recorded. After hotspots of the alignment start and stop positions were revealed, the flanking parts ( $>2 \mathrm{bp}$ ) were split out and converted to the sequences on the same strand. Gene fragments in these flanking parts were searched again using the BLASTX program with the default settings, which were more relaxed than those used in the previous searching. Both 5' and 3' flanking sequences were then aligned by MUSCLE v3.6, separately (Edgar, 2004).

The short reads were sorted into groups with respect to their alignment positions relative to the hotspots on the proteins. DNA secondary structures of representative short reads were constructed using the Mfold web server (Zuker, 2003). Default settings for folding temperature, window size and ionic conditions were employed. To calculate the free energy of all the short reads, UNAFold (Markham and Zuker, 2008) was used. The average and standard deviation of the free energy values were then calculated. To compare free energy of the short reads with the other reads in the metagenomes, long reads $>300$ bp were randomly truncated into short reads. Since the length of a DNA sequence is critical to the measurement of free energy, the average 
178 length of the short reads and random reads in a pairwise comparison should be similar. The 179 average lengths of the short reads for different genes ranged between 100 and $160 \mathrm{bp}$, and thus

180

181

182

183

184

185

186

187

188

189

190

191

192

193

194

195

196

197

198

199

200

random reads were further selected to generate four groups of random short reads, with an average length of 100, 120, 140 and 160 bp, respectively. Free energies of the short reads and the random sequences were compared by a Mann-Whitney test in a SPSS package (16.0).

The redundancy level of the reads belonging to different genes in Table 1 was checked by cdhit-454 (Niu et al., 2010). Similarity of matching parts in the reads was set at 97\%, lower than the threshold suggested by the pyrosequencing error rate, and then clusters among the reads were identified. During the check, long reads were retained in each cluster for further removal of redundancy. If at least $50 \%$ of a long read was covered by a short read, and if at least $95 \%$ of a short read could be aligned on a unique one, the two reads were clustered.

\section{Results}

\section{Short artifactual pyrosequencing reads}

We obtained 922,401, 480,994, 576,444, 489,923, and 1,099,605 raw pyrosequencing reads with an average length of 410, 382, 358, 397 and 402 bp, for Sed12, Sed63, Sed105, Sed183 and Sed222, respectively. Normally, the pyrosequencing platform produced reads in size of about 400 bp. Therefore, at least Sed63 and Sed105 metagenomes contained an unexpectedly high proportion of short reads. The distribution of all the pyrosequencing reads in different length ranges is shown in Fig. 2. A metagenome from the overlying brine water was used as the control to show over-abundant short reads in the sediment metagenomes. The short reads in Sed12 and Sed222 showed a similar distribution pattern to those in the control, whereas abnormally 
201 abundant short reads were observed in the other samples, such as those of 100-150 bp in Sed63,

202

203

204

205

206

207

208

209

210

211

212

213

214

215

216

217

218

219

220

221

222

223

of 50-160 bp in Sed105 and of 180-200 bp in Sed183 (Fig. 2). Thus, these reads might contain pyrosequencing artifacts .

To identify the artifacts, the short reads containing gene fragments were particularly focused on. By comparing the gene fragments in the reads with homologous genes, chimeras and rearrangements that possibly occurred during MDA might be revealed. Several genes were over-represented in the short reads. Fragments of K06988 gene (encoding a protein involved in utilization of DNA as a carbon source) and K07115 gene (encoding putative NADP oxidoreductase coenzyme) accounted for 40 and $37 \%$ of all the short reads of Sed63, respectively. The short reads in Sed105 were overwhelmed by K01440 gene fragments (encoding nicotinamidase), occupying $40 \%$ of its total short reads. About $9 \%$ of the short reads in Sed105 were assigned to K01409 gene (encoding O-sialoglycoproteinendopeptidase), the second most abundant gene. Fragments of K01627 gene (encoding KDO 8-P synthase) represented 37\% of the short reads in Sed222, although the distribution of Sed222 reads between 50-220 bp appeared to be normal (Fig. 2). For Sed12 and Sed183, none of the genes was as over-represented as those in the three samples mentioned above; yet 15\% of the short reads were assigned to K09705 gene (a hypothetical gene) in Sed183 as indicated by the abnormal bulge in 50-160 bp in Fig. 2.

The reads shorter than 220 bp were then counted for all the KEGG genes. With a cutoff of $\mathrm{P}_{<220}$ at 50\%, 11 more genes had abundant short reads (Table 1). The alignments of the reads with their KEGG homologous genes in Table 1 were deposited in the European Nucleotide Archive (http://www.ebi.ac.uk/ena/; accession: ERP002361). Overall, only one gene, K04567 (encoding 
224 lysyl-tRNA synthetase), was found for Sed12, but all the reads totaled 294. The genes, K07115, 225 K01440 and K01627, had a remarkably high $\mathrm{P}_{<220}(>98 \%)$. Statistical analysis was performed on 226 the length distribution of these genes by using a reference gene K00984, which had the most

227

228

229

230

231

232

233

234

235

236

237 abundant reads in all the samples and showed a normal length distribution (Fig. 3). When $\mathrm{N}_{<220}$ and $\mathrm{N}_{>220}$ were used to form a 2-way contingency table with the corresponding data of K00984, significantly higher numbers of reads were shorter than 220 bp for these genes $(P<0.0001)$. Under this criterion, there were many other genes also showing significant differences. The short reads for these genes were potentially artifactual reads if they were highly similar or concentrated in a small region of the corresponding genes.

Similarity between the reads for the over-represented genes was assessed by arranging them into clusters with a similarity cutoff of $97 \%$. Most of the reads, particularly with a high $\% \mathrm{~N}_{<220 \text {, were }}$ redundant. For example, 6180 reads for $\mathrm{K} 01627\left(\% \mathrm{~N}_{<220}=0.98\right)$ were sorted into 1189 clusters, and redundancy was up to $80 \%$. The ratio of cluster number to the total was negatively correlated to $\% \mathrm{~N}_{<220}(\mathrm{R}=0.77)$ (Table 1$)$. This means that most of the redundancy occurred in the short reads <220 bp. However, compared with other KEGG genes, the number of clusters is still large. This implies the presence of an abundance of sequence variants in the artifactual reads, which may have different sizes and internal polymorphisms.

\section{Artifactual reads were concentrated on a certain gene region}

More details regarding the length distribution of the short reads are provided. Taking the genes K07115, K01440, and K01627 as examples, the sizes of most of the short reads for K01440 in Sed105 ranged narrowly between 70 and 120 bp, whereas the length range of the short reads for 
K07115 and K06988 in Sed63 was widened to 70-160 and 110-220 bp, respectively (Fig. 3). The latter two also showed a sharp increase in the frequency of the short reads at ranges of 150-160 and 210-220 bp, respectively. The shortest average size of the short reads was 104 bp in K00257, with a standard deviation of 27 bp; the longest was $163 \mathrm{bp}$ in K07788 (Table 1). The length distribution of the reads for these genes was far from the expected pattern exemplified by the distribution of the reads for K00984 (Fig. 3).

Whether the artifactual reads were located at a certain gene region was examined. The aligned parts derived from BLASTX search were pin-pointed. Hotspots where alignment started and ended were recognized. For example, 117 aa, 131 aa and 186 aa in K06988 protein were the hotspots for the alignments between the artifactual reads and the protein (Fig. S1); 146 aa, 206 aa and 208 aa in K01627 protein were the most frequent points on which the alignment of the artifactual reads started or ended. Table S1 lists more such boundaries. Most of the short reads overlapped in the centre of the protein region and were thus confirmed to be artifacts. For the K06988 protein, the overlapping peaked at 160-170 aa, in which 89, 66, 50 and 56\% of the artifactual reads were located in Sed63, Sed105, Sed183, and Sed222, respectively (Fig. S2A). Therefore, the presence of the abundant artifactual reads for K06988 gene was not specific to one sediment layer. The artifactual reads (with $\mathrm{P}_{<220}>50 \%$ ) were found in all the samples except Sed12. There were only seven reads for the homolog in Sed12, and no peak was shown at the peaking range. The gene K00984 was taken as a control again because it did not have abnormally abundant reads in all the samples. The reads for all the homologs were evenly located on the protein and there were no notable abnormal distributions (Fig. S2B). 
270 The artifactual reads aligned to the highly covered K06988 protein region, i.e. 120-190 aa, were

271 examined in more detail. In Sed63, the reads were similar to the homolog Reut_B5423 from

272 Ralstonia eutropha. In this region, the similarity of the aligned regions was up to 69\% until the

273 end of the region (190 aa). The similarity at the boundary showed a sudden decline to about 50\%

274 (Fig. S3). This decline was negatively correlated with the change in read length. There was a

275 gradual decrease of average length of the reads in the protein region (Fig. S3). The highest

276 similarity corresponded with the lowest average read length of $260 \mathrm{bp}$. In comparison with the

277 other regions, the average length of the reads aligned to this region declined by $45 \%$. The two

278 ends of the alignments between the short reads and the protein encoded by Reut_B5423 were

279 somewhat highly concentrated at the hotspots. We pinpointed $47 \%$ of the alignment end

280 positions at 186 aa; 34\% of the start points were found at 117 aa and 131 aa (Fig. S1). It was also

281 true for the non-Sed12 samples in spite of their smaller read numbers for K06988. This result

282 further supports the presence of artifactual reads for the Reut_B5423 homolog.

283

284

285

286

287

288

289

290

291

292

\section{Stable secondary structures in genes and artifactual reads}

The characteristics of the read alignments with the proteins were indicative of special features in the corresponding regions in genes. DNA secondary structures were then examined in the subsections between the alignment hotspots in the artifactual reads. Three typical secondary structures were observed for the artifactual reads for K06988 gene (Fig. 4). At $37^{\circ} \mathrm{C}$, a high free energy of folding was observed in the reads with average dG equal to $-58.1,-39$ and -46.1 , respectively. Results showed that those started from 117 aa and 131 aa and could fold into a stable secondary structure with a long stem at the 5' end (Fig. 4A). On the other hand, the structures for those ending at the 186 aa were associated with a long stem at 3 ' end (Fig. 4C). 
293 However, the stable folding of the reads in Fig. 4A was completely attributable to the 294 introduction of an inserted fragment matched to the region of 117-131 aa. In BLASTX results, 295 the alignment of this fragment between 117 aa and 131 aa of the protein was, in fact, much 296 relaxed. The similarity at the DNA level was merely 53\% and almost none at protein level. 297 BLASTN search did not find similar sequences in the NCBI for this fragment in the read. 298 Actually, the part was a reverse complement of the downstream gene region starting from 131 aa 299 (Fig. 4A). It is worthwhile to note that the sequence 5' -TTTGCCGGCAAA-3' in Fig. 4A was a 300 small inverted repeat of itself and could introduce more complex structures. An additional 301 folding style was recognized in some reads for K06988. They were two merged gene fragments 302 but with a special arrangement of the fragments (Fig. 4B). The region ending at 186aa and 303 upstream were translocated upstream of 117 aa position. The translocation resulted in a more 304 stable structure with a dG of -66.95. The upper half of the stem was nearly identical to the 5' 305 stem in Fig. 4A, while the bottom half was the same as the one found at the 3' in Fig. 4C.

306 Likewise, the inserted fragment was along with its downstream sequence, indicating that this 307 unknown fragment had been integrated within the region around 131 aa before the translocation. 308 More variants were observed due to internal slippage regions up to $30 \mathrm{bp}$.

Moreover, the structure shown in Fig. 4C differs from that for the corresponding gene region 311 (Fig. S4). When the read and the gene were compared, several nucleotide replacements in the 312 read were found to make the secondary structure of the read much more stable. At the 5' end of 313 the read, four replacements were observed; at the 3' end, five replacements were found with four 314 Gs on the modified gene. 
316 The artifactual reads assigned to the other genes were also tested for free energy. Short reads

317 randomly trimmed down from long reads were used for a comparison. Results showed that free 318 energy of the artifactual reads for these genes was significantly lower than that of the random 319 reads of a similar size (U-test; $p<0.0001$ ) (Fig. 5). On the contrary, the reads belonging to 320 K00984 had even significantly higher free energy than the random reads (U-test; $p<0.0001$ ) 321 (Table S2).

322

\section{More translocation cases were frequently observed}

324 The frequency of the translocations occurred in the artifactual reads for K06988 gene was examined. There were about 125 artifactual reads whose secondary structures were clearly shown in Fig. 4B. Possibly, upstream and downstream regions in the models shown in Figs. 4A and 4C also contained the translocated fragments. They could not be recognized, possibly due to the settings of the BLASTX search. A total of 1554 artifactual reads for K06988 gene Reut_B5423 were collected, and the flanking regions ( $>2 \mathrm{bp}$ ) of the alignment hotspots were extracted. Of them, 272, 5' flanking sequences of 131 aa hotspot were recognized as the short relics between the 117 aa and 131 aa. These short flanking sequences with an average length of 12 bp would not form the 5' long stem as shown in Fig. 4A for most of the artifactual reads. In contrast, the 5' flanking sequences of 379 reads with an alignment start position at 117 aa were all matched to the upstream of 186 aa position. The extension to the upstream varied among the sequences and was, on average, 36 bp in size. A total of 937 reads were checked for the genes in their 3' flanking regions of 186 aa position (average length was $48 \mathrm{bp}$ ). Alignment of the sequences showed that they were at the downstream region of 117 aa position. This suggests 338

again that the unknown fragment corresponding to the region of 117-131 aa was a natural 
extension of the 5' of the 131 aa position. Overall, the translocation was detected in at least $60 \%$ of all the artifactual reads for the K06988 gene. The reads with alignment positions close to the hotspots were not taken into account, and therefore more such translocation events could be found in other reads. For K01627 genes in Sed222, at least 69\% of the 5,921 artifactual reads contained the translocation fragments. As such, the translocation as shown in Fig. 4B seems quite frequent in the artifactual reads. Difference between them under the stem-loop model was the size of the flanking regions.

\section{Chimeric gene fragments in short reads}

The translocated fragments in the artifactual reads were occasionally derived from different species. Chimeras were thus observed in the reads. Taking the artifactual reads for K06988 Reut_B5423 from Ralstonia as an example, we summarized the species with the best hits in BLASTX results for the 3' flanking fragments. Up to $83 \%$ resembled to the homologs in Cupriavidus necator N-1 (identity 56 - 62\%; positives 76 - 82\%). A few of the others clearly belonged to Methylobacterium species because the sequences were more similar to homologs from Methylobacterium than from other bacteria. Therefore, chimeras of gene fragments in the artifactual reads were confirmed.

To understand the formation of the chimeras, the artifactual reads for gene K01627 in Sed222 were further studied. Based on taxa of the best hits in BLASTX search, the K01627 genes could be assigned into three species: C. taiwanensis (47\%), Burkholderia ambifaria (41\%), and Variovorax paradoxus (12\%). A total of 338 artifactual reads contained chimeras, which were an integration of the homologs from B. ambifaria/V. paradoxus and that from $C$. taiwanensis. 
362

363

364

365

366

367

368

369

370

371

372

373

374

375

376

However, the chimeric phenomenon was not widespread because the artifactual reads for the other genes K07115, K01409, and K00257 did not contain obvious chimeric gene fragments from different species.

\section{Variants of the stem-loop model}

The folding structures of the artifactual reads and internal arrangement styles of the gene fragments were summarized; a schematic model was then proposed for individual genes. The stems for K06988 and K01627 reads along with three more under the schematic model are shown in Figure S5. The large part of the stem for K06988 was derived from 5' of the gene region, but that for K01627 was from 3' of the gene region. At the integration position, no large unknown fragments were inserted although the similarity between the reads and the genes at these positions was low. The central stems of three other genes including K07115, K01409 and K00257 had no insertions between the translocated fragments. Moreover, the stem might be shorter because the fragments adjacent to the integration sites were sometimes shorter than 10 bp, particularly for the shaded part in Figure S5. In case that the sequences by which the major part of the stem-loop was constructed, appeared in the flanking region, the size of the flanking sequences was generally longer to enable the conformation of the stem-loop. For the K01409 reads, the average size of the flanking sequences was $37 \mathrm{bp}$ for both ends; for the K07115 reads, it was 34 bp for 5' sequences and 29 bp for 3' sequences; for the K00257 reads, it was 22 and 24 bp for 5' and 3', respectively.

\section{Discussion}

In this study, artifactual pyrosequencing reads were uncovered in MDA-amplified sediment 
metagenomes. They were mostly redundant gene fragments, with stable secondary structures, translocations and chimeras. The translocated fragments belonged to neighboring parts of the homologous genes. A variety of strong DNA secondary structures were displayed in the reads, allowing us to propose a stem-loop model for interaction of the fragments. However, a fraction of these short reads $(<40 \%)$ could not fit the hypothetic model. This probably stemmed from the DNA extraction and pyrosequencing preparation, in which the artifactual fragments would be truncated. Furthermore, the artifactual reads for K01440 in Sed105 were likely generated by different means. The typical translocations were confirmed only in a low proportion of short reads; head-to-head translocated gene fragments in some reads could individually form a stem-loop structure. Chimeras were also observed in this study, but the formation mechanism might be different from a previous report (Lasken and Stockwell, 2007). In this study, the fragments in the chimeras were strictly matched to the neighboring gene regions, whereas the translocated fragments in the chimeras were separated by a genomic region of up to $10 \mathrm{~kb}$ in the other model.

There have been some early studies on several deep-sea sediment metagenomes (Biddle et al., 2008; Biddle et al., 2011; Inskeep et al., 2010; Quaiser et al., 2011), but a large number of short copies of gene fragments have never been reported. Researchers might have ignored the short DNA fragments in the samples, or did not use the same protocols as in this study. This study may shed some light on the process responsible for massive generation of these artifactual reads in the sediment metagenomes. The frequently observed translocations and secondary structures in the artifactual reads are the probable cause of the artifacts. They were not ascribed to 454 pyrosequencing because the artifactual reads generated by the 454 platform were identical and 
translocations in the artifacts have not been observed (Gomez-Alvarez et al., 2009). Moreover, the artifacts introduced by the 454 could affect many more genes, instead of the small number of genes in our metagenomes. Therefore, it is highly likely that the artifacts observed in this study were the result of MDA treatment. A study suggested that small DNA fragments with complex conformation will be amplified independently during MDA (Shoaib et al., 2008). Considering the high abundance of the artifactual reads in the metagenomes, these translocated DNA fragments with the stable secondary structure might have been more efficiently amplified in the MDA reaction. Additionally, we also noticed many nucleotide substitutions that made more pairings in the stems than in the genes. The substitutions may not occur on the genes, because the formation of strong stem-loop structures resulted from the substitutions might prohibit transcription. Instead, the MDA amplification of the artifactual reads could probably have created the nucleotide replacements which strengthened the secondary structures. The secondary structures could have first formed in the extracellular DNA, but might also originate from intracellular genomic DNA. In which steps the translocations occurred, is unknown at present, but the secondary structures might have been stabilized during the subsequent amplification.

At present, we do not yet have a satisfactory answer to the question of how the translocations happened and consequently got massively amplified. However, this study reminds us that DNA contamination, particularly extracellular DNA, in a sediment sample should be removed before MDA amplification and pyrosequencing. Otherwise, we suggested that short reads and usually abundant metagenomic reads ( $<220$ bp in this study) should be excluded from subsequent analyses. Finally, biological significance may be explored if the abundant short copies of gene fragments with stable structures persist in the metagenomes for pure unamplified DNA samples. 
437

438

439

440

441

442

443

444

445

446

447

448

449

450

451

452

453

454

455

456

457

458

459

460

461

462

463

464

465

466

467

468

Finally, we did not observe abundant artifactual reads in Sed12, although MDA amplification was also applied to the raw DNA sample. This seems exceptional to the observations in other sediment layers. Since this study is based on one metagenome from each layer, whether the abundant artifactual reads will repeatedly occur in the same genes from the same layer remains a question for future research.

\section{Acknowledgements:}

We thank Dr. Paul Harrison's comments to the manuscript.

\section{References}

Biddle JF, Fitz-Gibbon S, Schuster SC, Brenchley JE, House CH. 2008. Metagenomic signatures of the Peru Margin subseafloor biosphere show a genetically distinct environment. Proc Natl Acad Sci USA 105:10583-10588.

Biddle JF, White JR, Teske AP, House CH. 2011. Metagenomics of the subsurface Brazos-Trinity Basin (IODP site 1320): comparison with other sediment and pyrosequenced metagenomes. ISME J 5:1038-1047.

Borin S, Crotti E, Mapelli F, Tamagnini I, Corselli C, Daffonchio D. 2008. DNA is preserved and maintains transforming potential after contact with brines of the deep anoxic hypersaline lakes of the Eastern Mediterranean Sea. Saline Systems 4:10.

Bower AS. 2009. R/V Oceanus Voyage 449-6 Red Sea Atlantis II Deep Complex Area 19 October-1 November 2008. In: Woods Hole Oceanographic Institution.

Dean FB, Hosono S, Fang L, Wu X, Faruqi AF, Bray-Ward P, Sun Z, Zong Q, Du Y, Du J, Driscoll M, Song W, Kingsmore SF, Egholm M, Lasken RS. 2002. Comprehensive human genome amplification using multiple displacement amplification. Proc Natl Acad Sci USA 99:5261-5266.

Dean FB, Nelson JR, Giesler TL, Lasken RS. 2001. Rapid amplification of plasmid and phage DNA using phi29 DNA polymerase and multiply-primed rolling circle amplification. Genome Res 11:1095-1099.

Edgar RC. 2004. MUSCLE: multiple sequence alignment with high accuracy and high throughput. Nucl Acids Res 32:1792-1797.

Ferrer M, Werner J, Chernikova TN, Bargiela R, Fernández L, La Cono V, Waldmann J, Teeling H, Golyshina OV, Glöckner FO, Yakimov MM, Golyshin PN, The MSC. 2012. Unveiling microbial life in the new deep-sea hypersaline Lake Thetis. Part II: a metagenomic study. Environ Microbiol 14:268-281.

Gomez-Alvarez V, Teal TK, Schmidt TM. 2009. Systematic artifacts in metagenomes from complex microbial communities. ISME J 3:1314-1317. 
Huse S, Huber J, Morrison H, Sogin M, Welch D. 2007. Accuracy and quality of massively parallel DNA pyrosequencing. Genome Biol 8:R143.

Huse SM, Dethlefsen L, Huber JA, Welch DM, Relman DA, Sogin ML. 2008. Exploring microbial diversity and taxonomy using SSU rRNA hypervariable tag sequencing. PLoS Genetics 4:e1000255.

Inskeep WP, Rusch DB, Jay ZJ, Herrgard MJ, Kozubal MA, Richardson TH, Macur RE, Hamamura N, Jennings Rd, Fouke BW, Reysenbach A-L, Roberto F, Young M, Schwartz A, Boyd ES, Badger JH, Mathur EJ, Ortmann AC, Bateson M, Geesey G, Frazier M. 2010. Metagenomes from high-temperature chemotrophic systems reveal geochemical controls on microbial community structure and function. PLoS ONE 5:e9773.

Lasken R, Stockwell T. 2007. Mechanism of chimera formation during the Multiple Displacement Amplification reaction. BMC Biotechnol 7:19.

Levy-Booth DJ, Campbell RG, Gulden RH, Hart MM, Powell JR, Klironomos JN, Pauls KP, Swanton CJ, Trevors JT, Dunfield KE. 2007. Cycling of extracellular DNA in the soil environment. Soil Biol Biochem 39:2977-2991.

Logares R, Haverkamp THA, Kumar S, LanzÃ@n A, Nederbragt AJ, Quince C, Kauserud H. 2012. Environmental microbiology through the lens of high-throughput DNA sequencing: Synopsis of current platforms and bioinformatics approaches. J Microbiol Methods 91:106-113.

Markham NR, Zuker M. 2008. UNAFold: software for nucleic acid folding and hybridization. Methods Mol Biol. 453:3-31.

Niu B, Fu L, Sun S, Li W. 2010. Artificial and natural duplicates in pyrosequencing reads of metagenomic data. BMC Bioinformatics 11:187.

Pan X, Urban AE, Palejev D, Schulz V, Grubert F, Hu Y, Snyder M, Weissman SM. 2008. A procedure for highly specific, sensitive, and unbiased whole-genome amplification. Proc Natl Acad Sci USA 105:15499-15504.

Pietramellara G, Ascher J, Borgogni F, Ceccherini M, Guerri G, Nannipieri P. 2009. Extracellular DNA in soil and sediment: fate and ecological relevance. Biol Fertility Soils 45:219-235.

Quail M, Smith M, Coupland P, Otto T, Harris S, Connor T, Bertoni A, Swerdlow H, Gu Y. 2012. A tale of three next generation sequencing platforms: comparison of Ion Torrent, Pacific Biosciences and Illumina MiSeq sequencers. BMC Genomics 13:341.

Quaiser A, Zivanovic Y, Moreira D, Lopez-Garcia P. 2011. Comparative metagenomics of bathypelagic plankton and bottom sediment from the Sea of Marmara. ISME J 5:285-304.

Raghunathan A, Ferguson HR, Bornarth CJ, Song W, Driscoll M, Lasken RS. 2005. Genomic DNA amplification from a single bacterium. Appl Environ Microbiol 71:3342-3347.

Shoaib M, Baconnais S, Mechold U, Le Cam E, Lipinski M, Ogryzko V. 2008. Multiple displacement amplification for complex mixtures of DNA fragments. BMC Genomics 9:415.

Singh AH, Doerks T, Letunic I, Raes J, Bork P. 2009. Discovering functional novelty in metagenomes: Examples from light-mediated processes. J. Bacteriol. 191:32-41.

Smedile F, Messina E, La Cono V, Tsoy O, Monticelli LS, Borghini M, Giuliano L, Golyshin PN, Mushegian A, Yakimov MM. 2012. Metagenomic analysis of hadopelagic microbial assemblages thriving at the deepest part of Mediterranean Sea, Matapan-Vavilov Deep. Environ Microbiol In press. 


\section{(1)}

Spits C, Le Caignec C, De Rycke M, Van Haute L, Van Steirteghem A, Liebaers I, Sermon K. 2006. Optimization and evaluation of single-cell whole-genome multiple displacement amplification. Human Mutation 27:496-503.

Steinberger RE, Holden PA. 2005. Extracellular DNA in single- and multiple-species unsaturated biofilms. Appl Environ Microbiol 71:5404-5410.

Zhang K, Martiny AC, Reppas NB, Barry KW, Malek J, Chisholm SW, Church GM. 2006. Sequencing genomes from single cells by polymerase cloning. Nat Biotech 24:680-686.

Zuker M. 2003. Mfold web server for nucleic acid folding and hybridization prediction. Nucl. Acids Res. 31:3406-3415.

Table 1 Layer-specific overabundance of short reads for some genes. The KEGG genes in the table were abundant in short reads in sizes of $<220 \mathrm{bp}$ (the number is shown). The number of all the reads and percentage of the short reads $<220$ bp are shown for the individual genes. Lengths and $\mathrm{dG}$ values are given with $+/$ - standard deviation in the parentheses.

\begin{tabular}{llllllll}
\hline Sample & KEGG id & No. $<2$ 20bp & Total & \% N $<20$ & Clusters & $\begin{array}{l}\text { Average } \\
\text { length }\end{array}$ & $\begin{array}{l}\text { Average } \\
\text { dG }\end{array}$ \\
\hline Sed12 & K04567 & 161 & 294 & 55 & 237 & $162(33)$ & $-28.5(8.3)$ \\
Sed63 & K06988 & 2208 & 2435 & 91 & 648 & $162(35)$ & $-42.7(14.3)$ \\
& K07115 & 2056 & 2076 & 99 & 493 & $120(29)$ & $-28.0(10.3)$ \\
& K00859 & 140 & 192 & 73 & 63 & $161(41)$ & $-40.0(14.1)$ \\
& K02652 & 68 & 90 & 76 & 69 & $147(37)$ & $-38.3(13.5)$ \\
& K01061 & 60 & 79 & 76 & 63 & $147(37)$ & $-31.7(10.9)$ \\
Sed105 & K01440 & 2511 & 2528 & 99 & 462 & $109(23)$ & $-28.6(13.1)$ \\
& K01409 & 566 & 894 & 63 & 493 & $159(37)$ & $-38.5(12.9)$ \\
& K09800 & 405 & 461 & 88 & 277 & $149(37)$ & $-32.4(13.4)$ \\
& K01207 & 124 & 221 & 56 & 188 & $143(42)$ & $-24.8(9.2)$ \\
& K07788 & 68 & 100 & 68 & 85 & $163(40)$ & $-35.4(11.7)$ \\
& K00257 & 662 & 836 & 79 & 442 & $104(27)$ & $-26.3(8.7)$ \\
& K09705 & 289 & 301 & 96 & 135 & $115(21)$ & $-35.5(10.7)$ \\
& K00162 & 177 & 190 & 93 & 123 & $112(26)$ & $-28.0(7.8)$ \\
& K07506 & 121 & 155 & 78 & 109 & $143(27)$ & $-31.1(6.0)$ \\
& K01627 & 6068 & 6180 & 98 & 1189 & $143(38)$ & $-34.4(116.7)$ \\
& K01589 & 61 & 113 & 54 & 108 & $105(31)$ & $-25.6(11.4)$ \\
\hline
\end{tabular}

532

533

534

535

536

537

538 
Figure 2. Length range of the reads for five sediment samples. The control was the metagenome from the overlying Atlantis II brine water.

Figure 3. Length distribution of the reads for the genes with abundant short reads. Alignment positions of the reads on proteins were based on BLASTX results. The numbers in parentheses following the sample names are those of the short reads ( $<220 \mathrm{bp}$ ) and total reads.

Figure 4. Secondary structure of three representative reads for K06988 gene. Protein positions of K06988 gene are present on the reads. Length of read A is 152nt, and 8-151nt of this read was aligned to 117-164 aa of the K06988 protein. Length of read B is $210 \mathrm{nt}$ and 2-85 nt 
574

575 576 577

of this read was aligned to 159-186 aa of the protein; the region of 84-206 nt was aligned to $117-157$ aa. Length of read $\mathrm{C}$ is $179 \mathrm{nt}$, and $10-177 \mathrm{nt}$ of this read was aligned to 131-186 aa. The protein positions were indicated by arrows on the reads.

Figure 5. dG values of randomly-trimmed short reads from the metagenomes and those for selected KEGG genes. The names of the genes are shown beside the symbol of samples in which the average free energy was calculated for their reads. Symbols for the randomly-trimmed short reads in sizes of about 100, 120, 140 and 160 aa do not have a gene name beside them and were circled. 


\section{Figure 1}

fig1

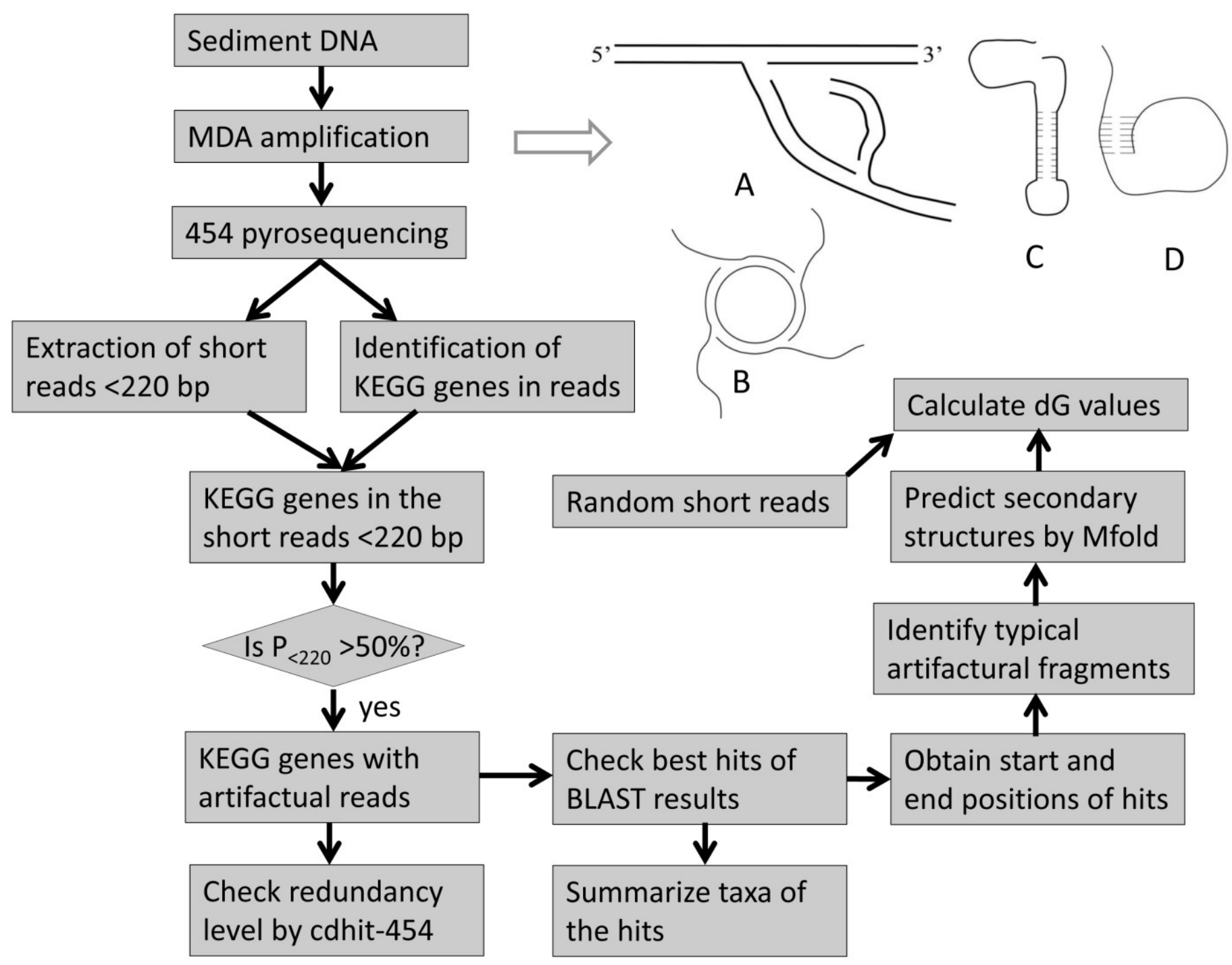




\section{Table $\mathbf{1}_{\text {(on next page) }}$}

fig2 


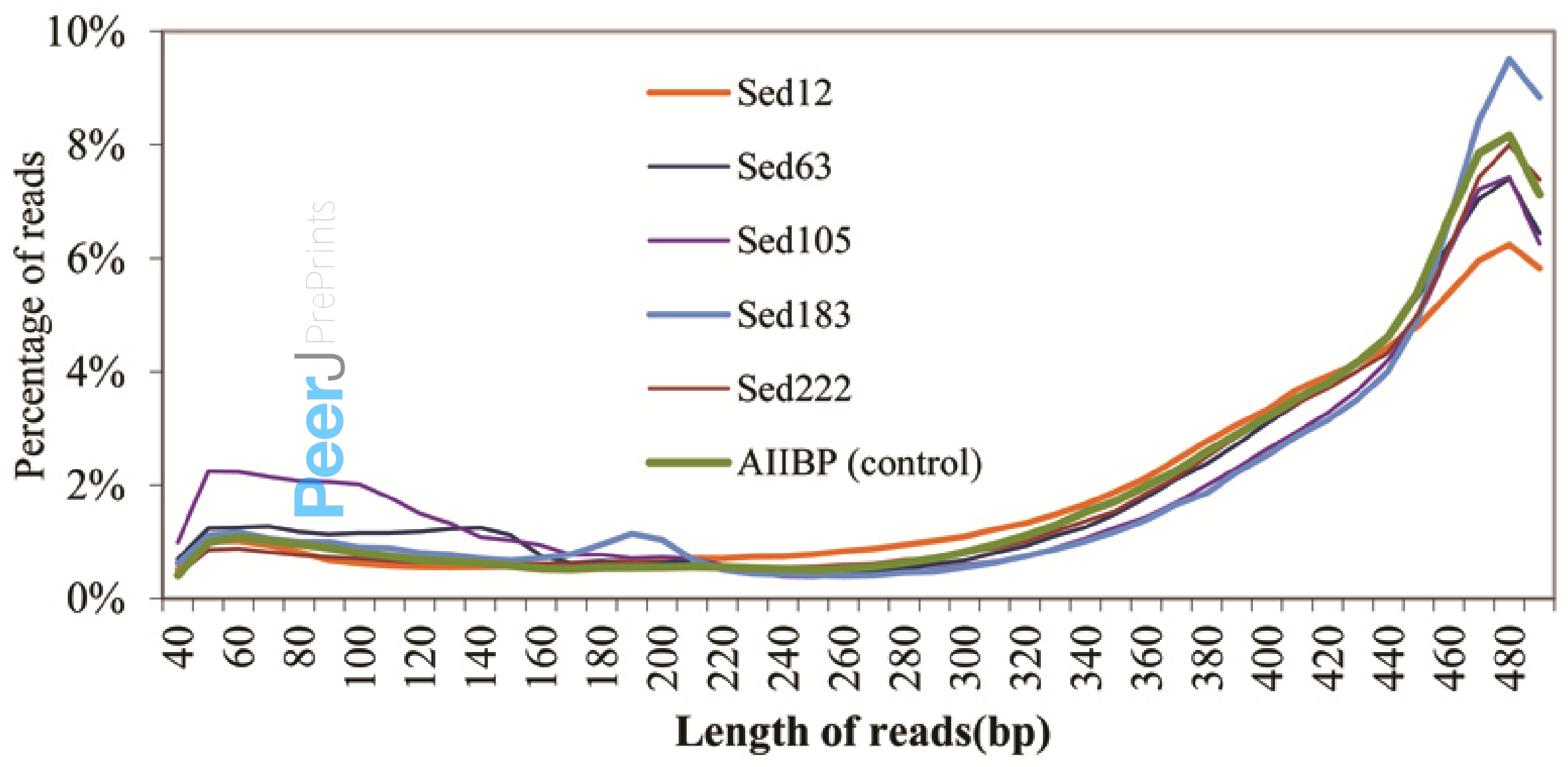




\section{Table 2 (on next page)}

fig3 


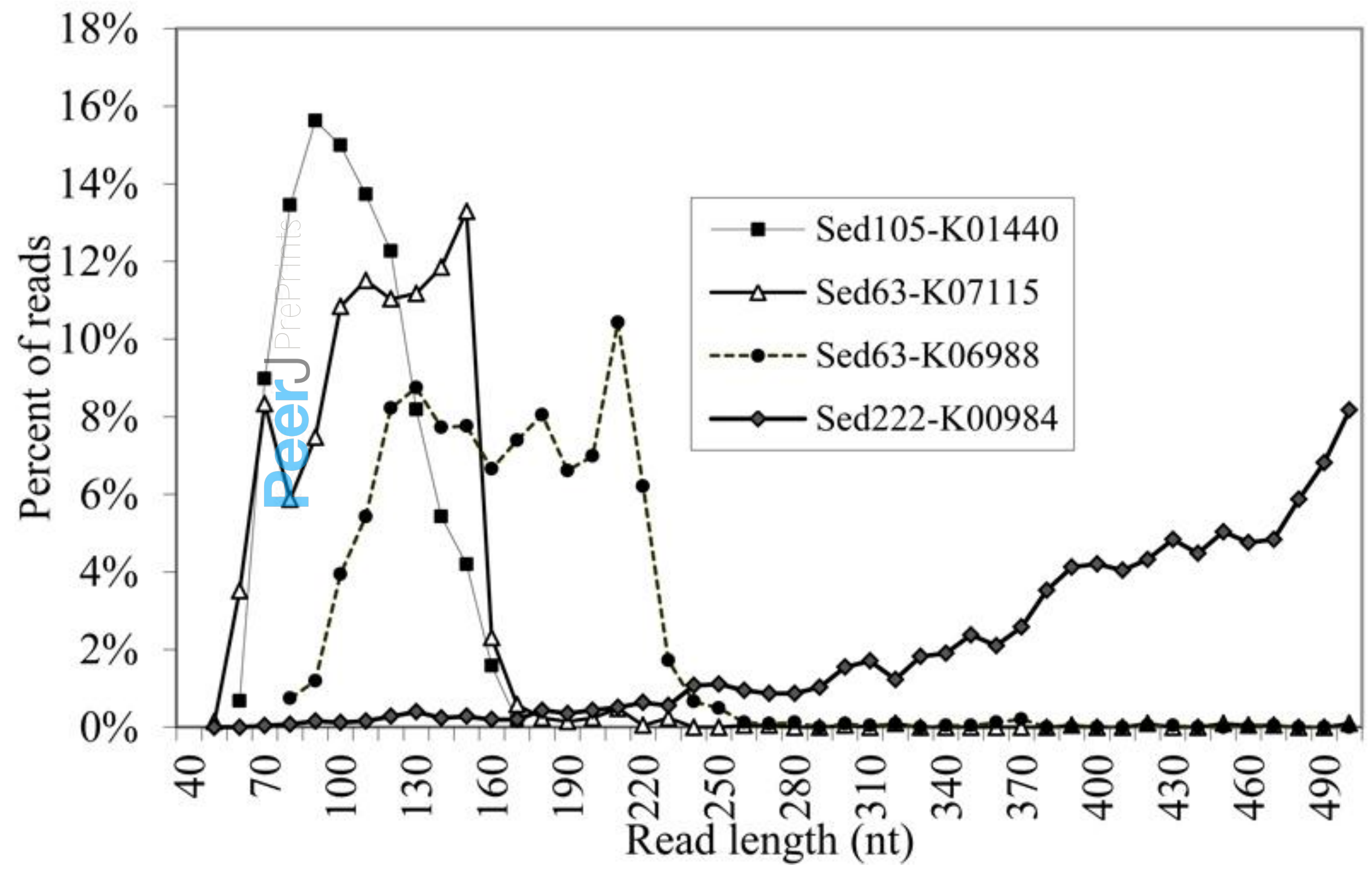




\section{Figure 2}

fig4
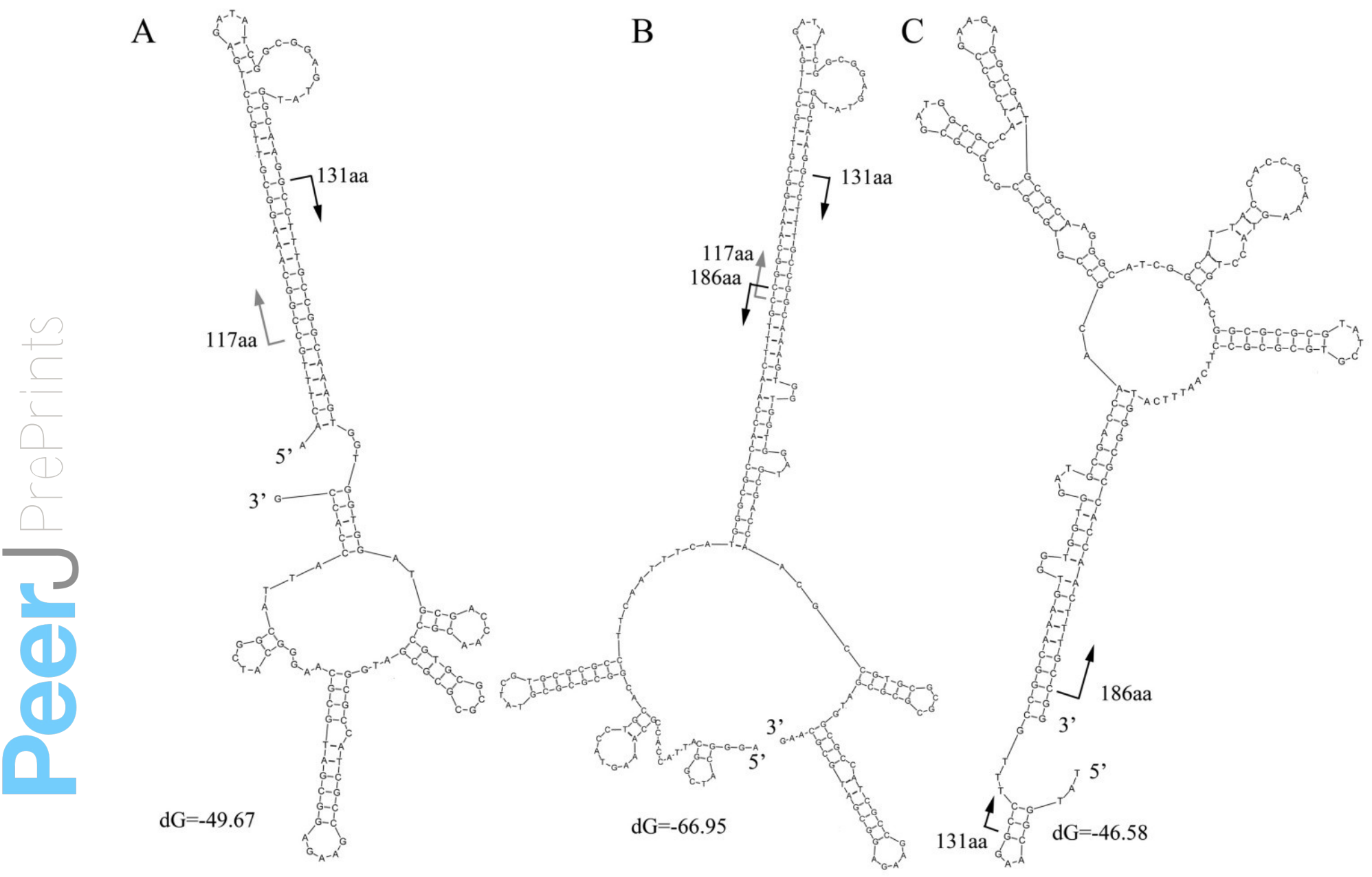


\section{Table 3 (on next page)}

fig5 


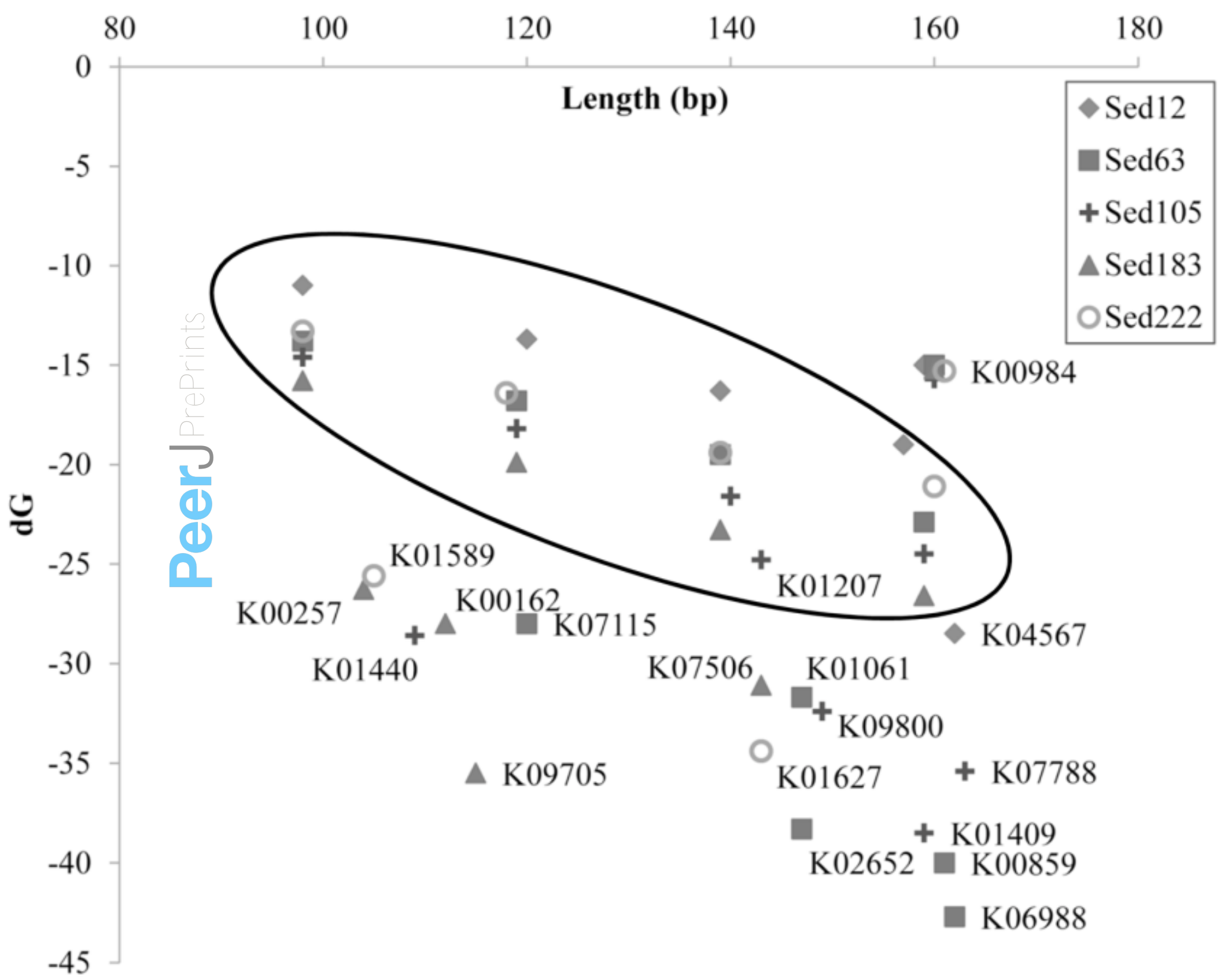

